\title{
Successful management of dyspnea in a patient with stiff person syndrome with noninvasive positive-pressure ventilation: A case report
}

\author{
${ }^{1}$ Du Hwan Kim $M D,{ }^{2}$ Min Cheol Chang $M D$ \\ ${ }^{1}$ Department of Physical Medicine and Rehabilitation, College of Medicine, Chung-Ang University, \\ Seoul; ${ }^{2}$ Department of Rehabilitation Medicine, College of Medicine, Yeungnam University, Daegu, \\ Republic of Korea
}

\begin{abstract}
Approximately half of patients with stiff person syndrome experienced dyspnea due to the involvement of the respiratory muscles. If these muscles are severely affected, respiratory failure can occur. Here, we describe a patient with stiff person syndrome whose nighttime dyspnea was successfully controlled by noninvasive positive-pressure ventilation (NIPPV). A 38-year-old female patient visited the emergency room due to nighttime dyspnea. Her $\mathrm{O}_{2}$ saturation was $93 \%$, and $\mathrm{PaCO}_{2}$ increased to $53.4 \mathrm{mmHg}$ on arterial blood gas analysis. No specific lesion was found on the chest radiograph. We considered that her dyspnea was caused by respiratory muscle stiffness following SPS. For the management of nighttime hypercapnia, we applied NIPPV with volume-targeted assist-control ventilation mode using an oronasal mask interface during sleep. After such treatment, $\mathrm{PaCO}_{2}$ was normalized, and nighttime dyspnea disappeared. We think that NIPPV would be a useful therapeutic option for managing dyspnea induced by stiff person syndrome.
\end{abstract}

Keywords: Stiff person syndrome; dyspnea; noninvasive positive-pressure ventilation; hypercapnia

\section{INTRODUCTION}

Stiff person syndrome (SPS) is a rare autoimmune disorder characterized by progressive muscle rigidity and stiffness of preferentially paraspinal and proximal limb muscles. ${ }^{1,2}$ The involuntary contraction of the agonist and antagonist muscles simultaneously occurs. ${ }^{3}$ It targets proteins associated with the gamma-aminobutyric acid (GABA)- or glycine-related inhibitory pathways in the spinal cord and brainstem. ${ }^{3}$ Among various possible clinical manifestations of SPS, respiratory symptoms develop in approximately 50\% of patients with SPS. ${ }^{4}$ According to the degree of respiratory muscle involvement, symptoms can be variously manifested from mild dyspnea to respiratory failure. For the treatment of symptoms following SPS, in mild cases, GABA receptor agonists, such as baclofen and benzodiazepines, can improve such symptoms. ${ }^{5}$ However, in severe cases, immune therapies with intravenous immunoglobulin, corticosteroids, rituximab, or long-term immunosuppressive agents are required ${ }^{5,6}$ If hypercapnic respiratory failure is prolonged, intubation and tracheostomy may be needed.

Noninvasive positive-pressure ventilation (NIPPV) is a respiratory technique of delivering mechanical ventilation without endotracheal intubation or tracheostomy. ${ }^{7}$ It is usually used for the treatment of hypoventilation in patients with several neuromuscular disorders, such as motor neuron disease, muscular dystrophy, and spinal cord injury. ${ }^{7}$ NIPPV can manage the symptoms caused by hypoventilation with avoiding tracheostomy.

In the current study, we present a patient with SPS whose dyspnea was successfully managed by NIPPV.

\section{CASE REPORT}

A year ago, a 38-year-old female patient with SPS visited the emergency room (ER) of a university hospital due to nighttime dyspnea with 7 (very hard breathing) of Rating of Perceived Dyspnea (RPD) (Table 1). Seven years ago, she was diagnosed with SPS based on the clinical findings of progressive sustained muscular stiffness of the bilateral proximal upper and lower

Address correspondence to: Min Cheol Chang, MD, Department of Physical Medicine and Rehabilitation, College of Medicine, Yeungnam University $317-1$, Daemyungdong, Namku, Taegu, 705-717, Republic of Korea. E-mail: wheel633@ynu.ac.kr

Date of Submission: 4 February 2021; Date of Acceptance: 19 February 2021

https://doi.org/10.54029/2021ehe 
Table 1: The Rating of Perceived Dyspnea scale

\begin{tabular}{ll}
\hline 0 & No shortness of breath at all \\
0.5 & Very, very slight shortness of breath \\
1 & Very mild shortness of breath \\
2 & Mild shortness of breath \\
3 & Moderate shortness of breath or breathing difficulty \\
4 & Somewhat severe shortness of breath \\
5 & Strong or hard breathing \\
6 & \\
7 & Severe shortness of breath or very hard breathing \\
8 & \\
9 & Extremely severe shortness of breath \\
10 & Shortness of breath so severe you need to stop the exercise or activity \\
\hline
\end{tabular}

limbs (trapezius, biceps, triceps, quadriceps, and hamstring muscles) and electromyographic findings of continuous motor unit activity in the agonist and antagonist muscles. Prior to this visit, the patient was having tolerable dyspnea with 2 (mild shortness of breathing) of RPD. Previously, several treatments, including oral medication (e.g., muscle relaxants and antispastic agents), local botulinum toxin injection, and intravenous immunoglobulin, were administered for the management of stiffness of the bilateral upper and lower limbs and mild dyspnea. However, these were only slightly effective for limb stiffness and not dyspnea. An intrathecal baclofen trial for limb stiffness was also performed. However, its effectiveness was only noted in the lower limbs, and therefore, intrathecal baclofen pump was not applied. While we continued conservative treatments for her limb stiffness, she developed severe nighttime dyspnea, daytime somnolence, fatigue, and headache a year ago.

Upon arrival at the ER, her $\mathrm{O}_{2}$ saturation was 93\%. Her other vital signs were as follows: blood pressure, $110 / 60 \mathrm{mmHg}$; pulse rate, 98 beats/ min; body temperature, $36.3{ }^{\circ} \mathrm{C}$; and respiratory rate, 16 breaths $/ \mathrm{min}$. Her initial laboratory test results from the ER were as follows: white blood cell count, $6780 / \mu \mathrm{L}$; C-reactive protein level, $0.6 \mathrm{mg} / \mathrm{L}$; creatinine kinase, $234 \mathrm{U} / \mathrm{L}$ (normal: 32-211); and, for the arterial blood gas analysis (ABGA), pH, 7.458; $\mathrm{PaO}_{2}, 89.3 \mathrm{mmHg} ; \mathrm{PaCO}_{2}$, $53.4 \mathrm{mmHg} ; \mathrm{HCO}_{3}, 37.8 \mathrm{mmol} / \mathrm{L}$; and $\mathrm{SaO}_{2}$, $96.6 \%$. The chest radiograph showed no specific abnormal findings. At the initial ER visit, she was discharged without specific managements except for 1-L $\mathrm{O}_{2}$ supply because her symptoms were slowly improving at daytime. However, after discharge from ER, she had experienced recurrent nighttime dyspnea and daytime somnolence.
One month after this, she was admitted to the department of physical medicine and rehabilitation for the management of recurrent nighttime dyspnea. The pulmonary function test showed decreased forced vital capacity (FVC, $57 \%$ of the predicted value). Nighttime ABGA revealed a $\mathrm{PaCO}_{2}$ of $52.2-58.3 \mathrm{mmHg}$, indicating hypercapnia, but daytime $\mathrm{ABGA}$ showed a $\mathrm{PaCO}_{2}$ of $42.6-50.0 \mathrm{mmHg}$. In the ultrasound (US) examination, hyperechogenicity of the bilateral biceps, intercostalis, and diaphragm muscles was observed and could suggest myositis in these muscles (Figure 1). We considered that the patient's nighttime dyspnea was induced by stiffness of the respiratory muscles following SPS on the basis of her medical history and results of chest radiograph and US. For the management of nighttime hypercapnia, we applied NIPPV with volume-targeted assist-control ventilation mode using an oronasal mask interface during sleep.

The parameters were adjusted according to the patient's response and end-tidal $\mathrm{CO}_{2}$ monitoring. During the NIPPV application, $\mathrm{PaCO}_{2}$ lowered to $42.0 \mathrm{mmHg}$, and nighttime dyspnea disappeared. At discharge, the setting parameters were as follows: respiration rate, 16 breaths/min; tidal volume, $530 \mathrm{~mL}$; positive end-expiratory pressure, $5 \mathrm{cmH}_{2} \mathrm{O}$; inspiration time, $1.1 \mathrm{~s}$; and $\mathrm{FiO}_{2}, 0.21$. During the continuous monitoring for 1 year, she responded well to the NIPPV application with adjustment of tidal volume between 500 and 550 $\mathrm{mL}$. At 1-year follow-up after the treatment, the patient reported that she had continuously used NIPPV during sleep at night and her nighttime dyspnea improved to 1 (very mild shortness of breath) of RPD. Her limbs muscle stiffness remained unchanged, but daytime somnolence, fatigue, and headache improved subjectively. 


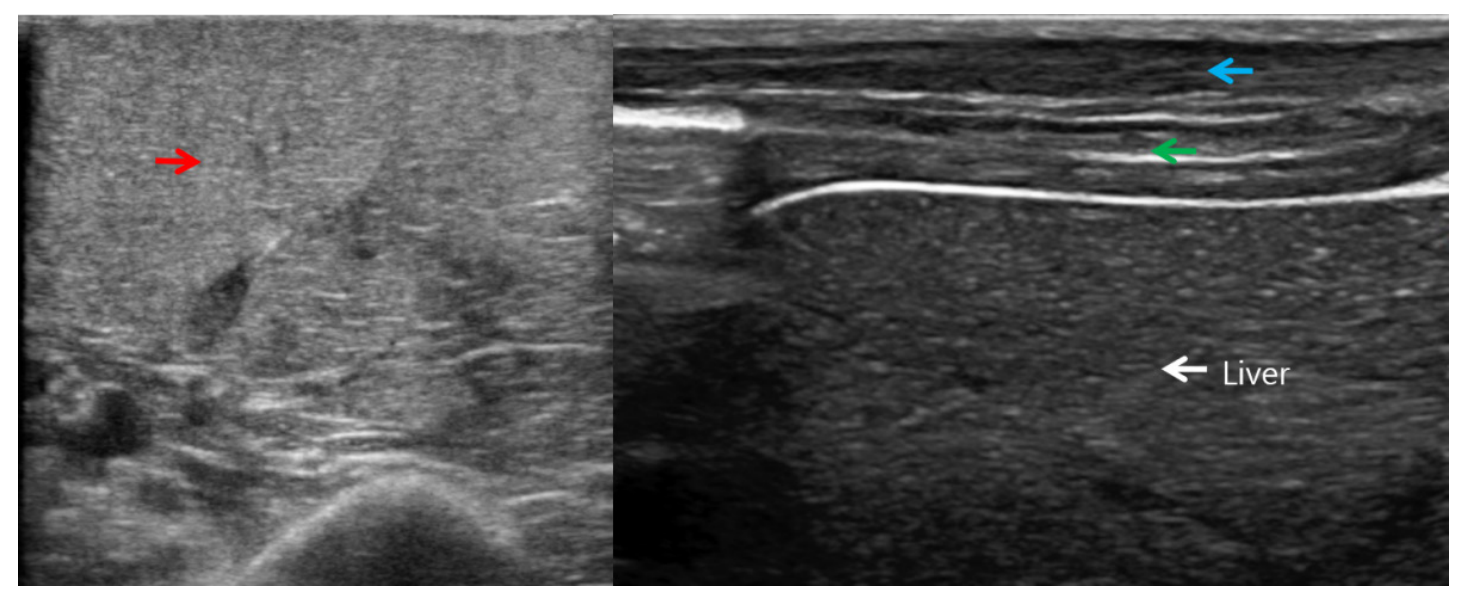

Figure 1. The findings of ultrasound examination. Hyperechogenicity of the bilateral biceps (red arrow), intercostalis (blue arrow), and diaphragm muscles (green arrow) was revealed.

\section{DISCUSSION}

In this case report, we describe a patient with nighttime dyspnea due to SPS who exhibited a successful response to the NIPPV application. Our patient's nighttime dyspnea and hypercapnia during sleep disappeared after such treatment.

It is suggested that thoracic cage constriction by rigidity or spasm of the trunk muscles is the primary cause of dyspnea in patients with SPS. ${ }^{1,2}$ Also, diaphragmatic involvement may contribute to the development of respiratory symptoms in these patients. ${ }^{4}$ This respiratory muscle involvement causes lung disease with a restrictive pattern. In 2018, Allen et al.$^{8}$ evaluated the presence of dyspnea and associated pulmonary function abnormalities in 31 patients with SPS. Although approximately half of patients with SPS are known to have dyspnea and associated pulmonary function abnormalities, in their study, 29 of these patients with SPS had dyspnea. Moreover, the mean FVC was $76.5 \%$ of the predicted value, which is indicative of lung disease with a restrictive pattern. In our patient, nighttime dyspnea developed 7 years after the diagnosis of SPS. Despite various treatments, including oral medications, local botulinum toxin injection, and intravenous immunoglobulin, the patient's respiratory symptom was not satisfactorily managed.

NIPPV is a safe and noninvasive tool for managing acute or chronic respiratory failures in patients with restrictive lung disease caused by various neuromuscular disorders whose bulbar function is intact. ${ }^{7}$ It can be used for prolonged ventilation with favorable outcomes, even in cases with severe restrictive lung disease.
Furthermore, several previous studies showed that various respiratory symptoms, such as dyspnea, hypoventilation, nocturnal sleep disruption, headache, and daytime hypersomnolence, were improved after applying NIPPV. ${ }^{7,9}, 10$ Accordingly, we proposed that the NIPPV application would also effectively manage dyspnea in our patient. As a result of such treatment, $\mathrm{PaCO}_{2}$ decreased, and the patient's nighttime dyspnea disappeared. In addition, NIPPV could effectively control dyspnea even prolonged use of 1 year avoiding tracheostomy. In clinical practice, dyspnea in patients with SPS has been managed by muscle relaxants, antispastic agents, rituximab, intravenous immunoglobulin, and plasmapheresis. ${ }^{5,6}$ Our study showed that NIPPV can effectively manage dyspnea induced by SPS. Furthermore, it can be safely applied.

Additionally, as reported in the previous study, our patient had focal myositis in the bilateral proximal muscles of the upper and lower limbs. ${ }^{11}$ In the US examination performed during admission for the NIPPV application, hyperechogenicity was observed in the intercostalis and diaphragm muscles as well as bilateral biceps. We think that these US findings may indicate the presence of myositis in these muscles. ${ }^{12}$ Also, although the creatinine kinase level was slightly elevated, such result supports our assumption that myositis may be present in the respiratory muscles. Myositis is thought to be caused by sustained muscle contraction. ${ }^{11}$ However, further investigations such as muscle biopsy for diaphragm were not done. It could still be due to polymyositis because there are a few reports that SPS and polymyositis may co-exist. ${ }^{13,14}$ 
In the current study, we reported a patient with SPS whose nighttime dyspnea was successfully managed by the NIPPV application. To the best of our knowledge, this is the first study to show that NIPPV can be one of the useful therapeutic options for managing dyspnea induced by SPS. However, because this is a case report, our study is limited. Also, we still do not know when is the optimal time to start NIPPV treatment in patients with dyspnea after SPS. Further studies involving larger cases would be warranted for wide NIPPV application for controlling respiratory symptoms in patients with SPS. Also, studies for determining the optimal time to initiate NIPPV treatment should be conducted in the future.

\section{DISCLOSURE}

Financial support: None

Conflict of interest: None

\section{REFERENCES}

1. Baizabal-Carvallo JF, Jankovic J. Stiff-person syndrome: insights into a complex autoimmune disorder. J Neurol Neurosurg Psychiatry 2015;86(8):840-8.

2. Balint B, Bhatia KP. Stiff person syndrome and other immune-mediated movement disorders - new insights. Curr Opin Neurol 2016;29(4):496-506.

3. Dalakas MC, Jujii M, Li M, et al. The clinical spectrum of anti-GAD antibody-positive patients with stiff-person syndrome. Neurology 2000;55(10):15315.

4. Sexauer W, Woodford M, Pack K, et al. Dyspnea in patients with stiff-person syndrome. Am J Med Sci 2019;358(4):268-72.

5. Albahra S, Yates SG, Joseph D, et al. Role of plasma exchange in stiff person syndrome. Transfus Apher Sci 2019;58(3):310-2.

6. Rineer S, Fretwell T.Evaluation of treatment outcomes in patients with stiff person syndrome with rituximab vs. standard of care. Cureus 2017;9(6):e1387.

7. Chong HS, Padua MR, Kim JS, et al. Usefulness of noninvasive positive-pressure ventilation during surgery of flaccid neuromuscular scoliosis. $J$ Spinal Disord Tech 2015;28(8):298-300.

8. Allen A, Rakocevic G, Woodford M, et al, Unrecognized respiratory manifestations of stiff person syndrome (SPS). Neurology 2018; 90 (15 Supplement): P5.463.

9. Comellini V, Pacilli AMG, Nava S. Benefits of noninvasive ventilation in acute hypercapnic respiratory failure. Respirology 2019;24(4):308-17.

10. Park D, Lee GJ, Kim HY, et al. Different characteristics of ventilator application between tracheostomy- and noninvasive positive pressure ventilation patients with amyotrophic lateral sclerosis. Medicine (Baltimore) 2017;96(10):e6251.

11. No SW, Im IK, Kim DH. Stiff person syndrome with evidence of nonspecific focal myositis secondary to sustained muscle contraction: A case report. $P M R$ 2018;10(12):1426-30.

12. Mul K, Horlings CGC, Vincenten SCC, et al. Quantitative muscle MRI and ultrasound for facioscapulohumeral muscular dystrophy: complementary imaging biomarkers. J Neurol 2018;265(11):2646-55.

13. Dalakas MC, Fujii M,Li M, McElroy B. The clinical spectrum of anti-GAD antibody-positive patients with stiff-person syndrome. Neurology 2000;55(10): 15315.

14. Pakeerappa PN, Birthi P, Salles S. Botulinum toxin A injection to facial and cervical paraspinal muscles in a patient with stiff person syndrome: A case report. $P M$ $R$ 2015;7(3):326-8. 